\title{
Clinical picture and risk prediction of short-term mortality in cardiogenic shock
}

\author{
Veli-Pekka Harjola ${ }^{1 *, \dagger}$, Johan Lassus ${ }^{2 \dagger}$, Alessandro Sionis ${ }^{3}$, Lars Køber ${ }^{4}$, \\ Tuukka Tarvasmäki ${ }^{5}$, Jindrich Spinar ${ }^{6}$, John Parissis ${ }^{7}$, Marek Banaszewski ${ }^{8}$, \\ Jose Silva-Cardoso9, Valentina Carubelli10, Salvatore Di Somma ${ }^{11}$, Heli Tolppanen², \\ Uwe Zeymer ${ }^{12}$, Holger Thiele ${ }^{13}$, Markku S Nieminen ${ }^{2}$, and Alexandre Mebazaa ${ }^{14}$, \\ for the CardShock study investigators and the GREAT network
}

\begin{abstract}
${ }^{1}$ Emergency Medicine, University of Helsinki, Helsinki University Hospital, Helsinki, Finland; ${ }^{2}$ Cardiology, University of Helsinki, Heart and Lung Center, Helsinki University Hospital, Helsinki, Finland; ${ }^{3}$ Intensive Cardiac Care Unit, Cardiology Department, Hospital de la Santa Creu i Sant Pau, Biomedical Research Institute Sant Pau (IIB Sant Pau), Barcelona, Spain; ${ }^{4}$ Rigshospitalet, Copenhagen University Hospital, Division of Heart Failure, Pulmonary Hypertension and Heart Transplantation, Copenhagen, Denmark; ${ }^{5}$ Emergency Medicine, University of Helsinki, Helsinki University Hospital, Helsinki, Finland; ${ }^{6}$ University Hospital Brno, Department of Internal Medicine and Cardiology, Brno, Czech Republic; ${ }^{7}$ Attikon University Hospital, Heart Failure Clinic and Secondary Cardiology Department, Athens, Greece; ${ }^{8}$ Institute of Cardiology, Intensive Cardiac Therapy Clinic, Warsaw, Poland; ' ${ }^{9}$ niversity of Porto, CINTESIS, Department of Cardiology, Porto Medical School, São João Hospital Center, Porto, Portugal; ${ }^{10}$ Division of Cardiology, Department of Medical and Surgical Specialties, Radiological Sciences, and Public Health, University and Civil Hospital of Brescia, Italy; ${ }^{11}$ Department of Medical Sciences and Translational Medicine, University of Rome Sapienza, Emergency Medicine Sant'Andrea Hospital, Rome, Italy; ${ }^{12}$ Klinikum Ludwigshafen, Medizinische Kinik B, Ludwighafen, Germany; ${ }^{13}$ University of Lübeck, Medical Clinic II, Cardiology, Angiology and Intensive Care, Lübeck, Germany; and ${ }^{14}$ INSERM U942, Hopital Lariboisiere, APHP and University Paris Diderot, Paris, France
\end{abstract}

Received 11 November 2014; revised 12 January 2015; accepted 23 January 2015; online publish-ahead-of-print 28 March 2015

Aims

The aim of this study was to investigate the clinical picture and outcome of cardiogenic shock and to develop a risk prediction score for short-term mortality.

Methods

and results

The CardShock study was a multicentre, prospective, observational study conducted between 2010 and 2012. Patients with either acute coronary syndrome (ACS) or non-ACS aetiologies were enrolled within $6 \mathrm{~h}$ from detection of cardiogenic shock defined as severe hypotension with clinical signs of hypoperfusion and/or serum lactate $>2 \mathrm{mmol} / \mathrm{L}$ despite fluid resuscitation ( $n=219$, mean age $67,74 \%$ men). Data on clinical presentation, management, and biochemical variables were compared between different aetiologies of shock. Systolic blood pressure was on average $78 \mathrm{mmHg}$ (standard deviation $14 \mathrm{mmHg}$ ) and mean arterial pressure $57(11) \mathrm{mmHg}$. The most common cause $(81 \%)$ was ACS (68\% ST-elevation myocardial infarction and $8 \%$ mechanical complications); $94 \%$ underwent coronary angiography, of which $89 \% \mathrm{PCl}$. Main non-ACS aetiologies were severe chronic heart failure and valvular causes. In-hospital mortality was $37 \%(n=80)$. ACS aetiology, age, previous myocardial infarction, prior coronary artery bypass, confusion, low LVEF, and blood lactate levels were independently associated with increased mortality. The CardShock risk Score including these variables and estimated glomerular filtration rate predicted in-hospital mortality well (area under the curve 0.85 ).

Conclusion Although most commonly due to ACS, other causes account for one-fifth of cases with shock. ACS is independently associated with in-hospital mortality. The CardShock risk Score, consisting of seven common variables, easily stratifies risk of short-term mortality. It might facilitate early decision-making in intensive care or guide patient selection in clinical trials.

Trial NCT01374867.

registration

Keywords 


\section{Introduction}

Cardiogenic shock is a severe state of systemic hypoperfusion due to cardiac dysfunction, often resulting in multiorgan failure. The mortality, both in-hospital and overall, is unacceptably high.

Since the majority of cardiogenic shock is caused by acute myocardial infarction (MI), most of the data are derived from registries of patients with $\mathrm{MI}$. However, only $5-8 \%$ of patients in these registries present with shock. ${ }^{1-4}$ Almost two decades have passed since the completion of the SHOCK trial, the landmark trial that established the role of early coronary revascularization in MI patients with shock. ${ }^{5}$ Subsequently, thrombolytic therapy and coronary artery bypass grafting (CABG) have been replaced by primary $\mathrm{PCl}$ as the first-line management. ${ }^{6-8}$ Recent reports on improving outcome in cardiogenic shock may reflect this change in management strategy. ${ }^{2,3}$

In the recently published IABP-SHOCK II, the largest randomized trial in cardiogenic shock to date, only patients with MI were included. ${ }^{9}$ Though several cardiac emergencies other than acute coronary syndromes (ACS) may cause shock, contemporary data on unselected patients with cardiogenic shock are lacking. ${ }^{10,11}$ Accordingly, analysis of the clinical presentation and management of patients with cardiogenic shock in the modern era is necessary for development of new therapeutic approaches and for more accurate risk stratification in this complex syndrome. Moreover, there is an obvious clinical need for a simple tool for risk prediction to aid in the urgent and critical task of selecting proper management, including targeting the most appropriate candidates for advanced therapies. Besides, a risk classification tool would be valuable for including more homogenous patient populations in clinical trials as well as for assessing the case mix in different hospitals in order to carry out proper benchmarking and epidemiological evaluations.

Therefore, we conducted a prospective, observational multicentre and multinational study, including the whole spectrum of aetiologies of cardiogenic shock. The aim of the CardShock study was to investigate the aetiology, clinical and biochemical characteristics, as well as management and prognosis of this medical emergency. In particular, we sought to recognize potential differences in clinical picture and outcome between patients with and without ACS as aetiology, and to develop a risk prediction tool for mortality in cardiogenic shock that could be applied shortly after presentation in the emergency department or hospital admission.

\section{Methods}

The CardShock study (NCT01374867 at www.clinicaltrials.gov) is a European prospective, observational multicentre and multinational study on cardiogenic shock. Patients were recruited between October 2010 and 31 December 2012 from emergency departments, cardiac and intensive care units, as well as catheter laboratories in nine tertiary hospitals from eight countries.

\section{Inclusion criteria and data collection}

The study enrolled consecutive patients aged over 18 years within $6 \mathrm{~h}$ from identification of cardiogenic shock. In addition to an acute cardiac cause, the inclusion criteria required systolic blood pressure to be $<90 \mathrm{mmHg}$ (after adequate fluid challenge) for $30 \mathrm{~min}$ or there to be a need for vasopressor therapy to maintain systolic blood pressure $>90 \mathrm{mmHg}$ and signs of hypoperfusion (altered mental status/confusion, cold periphery, oliguria $<0.5 \mathrm{~mL} / \mathrm{kg} / \mathrm{h}$ for the previous $6 \mathrm{~h}$, or blood lactate $>2 \mathrm{mmol} / \mathrm{L}$ ). Exclusion criteria were shock caused by ongoing haemodynamically significant arrhythmias or after cardiac or non-cardiac surgery.

The aetiology of cardiogenic shock was determined by local investigators. ACS aetiology was defined as shock caused by MI [with (STEMI) or without ST-elevation (non-STEMI)]. Echocardiography was performed per protocol at study entry. Creatinine, C-reactive protein (CRP), high-sensitivity troponin T (hsTnT), and NT-proBNP (Roche Diagnostics, Basel, Switzerland) were analysed centrally from blood samples stored at $-80^{\circ} \mathrm{C}$. Arterial blood lactate and $\mathrm{pH}$ were analysed locally. Estimated glomerular filtration rate (eGFR) was calculated from creatinine values using the CKD-EPI (Chronic Kidney Disease Epidemiology Collaboration) equation. ${ }^{12}$ Patients were treated according to local practice, and treatment and procedures were registered. The primary endpoint was all-cause in-hospital mortality. Vital status during follow-up was determined through direct contact with the patient or next of kin, or through population and hospital registers. The study was approved by local ethics committees and conducted in accordance with the Declaration of Helsinki.

\section{Statistical analysis}

Results are presented as numbers ( $n$ ) and percentages (\%), means and standard deviations (SD), or median and interquartile range (IQR) for variables with a skewed distribution. Between-group comparisons were performed using Student's $t$-test or Mann-Whitney U-test, as appropriate. Differences in mortality were assessed by $\chi^{2}$ or by drawing Kaplan-Meier survival curves which then were compared with the log-rank test.

Logistic regression analysis was used to identify variables associated with in-hospital mortality. Based on significant association with outcome on univariate analysis (with a $P$-value $<0.2$ accepted for retention), multivariable logistic regression modelling was performed. Age, gender, and a variable accounting for differences between centres were always included in the multivariable models. Independent predictors of in-hospital mortality were identified through multiple testing. Variables with $P>0.1$ in adjusted analyses were not retained in the final model. Goodness-of-fit was assessed by the Hosmer-Lemeshow test. Results from the regression analyses are presented as odds ratios (ORs) with 95\% confidence intervals $(\mathrm{Cls})$.

A risk prediction model for in-hospital mortality was constructed based on variables independently associated with all-cause death in the CardShock study. The discriminative ability of the risk prediction model was assessed by the area under the receiver operating characteristic (ROC) curve (AUC) or c-statistic. The performance of the prediction model and the final CardShock risk Score were then compared with a previously published scoring system from the SHOCK trial $^{13}$ and APACHE II score, ${ }^{14}$ and further validated in an external cohort $(n=384)$ from the IABP-SHOCK II trial (see also Supplementary material online, Appendix 1). ${ }^{9}$

Finally, seven categorical parameters were included in the CardShock risk Score, giving a maximum of nine points. The distribution of the population and predicted and observed mortality within risk categories were calculated. A two-sided $P$-value $<0.05$ was regarded as statistically significant. SPSS 21.0 statistical software (IBM Corp, Armonk, NY, USA) was used in all statistical analyses. 
Table 1 Baseline characteristics of the study population

\begin{tabular}{|c|c|c|c|c|}
\hline Characteristic & All $(n=219)$ & $\operatorname{ACS}(n=177)$ & Non-ACS $(n=42)$ & $P$-value \\
\hline Age, years & $67(12)$ & $68(11)$ & $62(15)$ & 0.03 \\
\hline Age > 75 years, $n(\%)$ & $54(25)$ & $45(25)$ & $9(21)$ & 0.6 \\
\hline Women, $n(\%)$ & $57(26)$ & $39(22)$ & $18(43)$ & 0.006 \\
\hline BMI, median (IQR) & $26.5(24.2-29.0)$ & $26.6(24.2-29.0)$ & $25.8(23.0-29.4)$ & 0.3 \\
\hline \multicolumn{5}{|l|}{ Medical history, n (\%) } \\
\hline Coronary artery disease & $76(35)$ & $59(334)$ & $17(40)$ & 0.4 \\
\hline Previous myocardial infarction & $54(25)$ & $42(24)$ & $12(29)$ & 0.5 \\
\hline \multicolumn{5}{|l|}{ Prior revascularization } \\
\hline $\mathrm{PCl}$ & $32(15)$ & $28(16)$ & $4(10)$ & 0.3 \\
\hline CABG & $16(7)$ & $10(6)$ & $6(14)$ & 0.05 \\
\hline Heart failure & $36(16)$ & $16(9)$ & $20(48)$ & $<0.001$ \\
\hline Hypertension & $132(60)$ & $111(63)$ & $21(50)$ & 0.13 \\
\hline Diabetes & $62(28)$ & $56(32)$ & $6(14)$ & 0.03 \\
\hline Asthma/COPD & $25(11)$ & $18(10)$ & $7(17)$ & 0.2 \\
\hline Renal insufficiency & $25(11)$ & $14(8)$ & $11(26)$ & 0.001 \\
\hline Atrial fibrillation & $32(15)$ & $17(10)$ & $15(36)$ & $<0.001$ \\
\hline Stroke/TIA & $20(9)$ & $14(8)$ & $6(14)$ & 0.2 \\
\hline Smoker & $87(40)$ & $78(44)$ & $9(21)$ & 0.01 \\
\hline
\end{tabular}

\section{Results}

\section{Study population}

A total of 219 patients were included in the study. Twenty-four per cent of them had shock at presentation to hospital, whereas $62 \%$ developed shock within the first $24 \mathrm{~h}$ from admission. Only $14 \%$ developed shock after $24 \mathrm{~h}$ from admission.

Baseline characteristics are described in Table 1. Briefly, mean age was 67 (12) years, and 74\% were men. The main co-morbidities were hypertension $(60 \%)$, CAD $(35 \%)$, and diabetes $(28 \%)$, while a history of previous MI (25\%) or heart failure (16\%) was less common. At detection of shock, systolic blood pressure was on average 78 (14) $\mathrm{mmHg}$, diastolic blood pressure 47 (10) $\mathrm{mmHg}$, mean arterial pressure $57(11) \mathrm{mmHg}$, and heart rate 90 (28) b.p.m. Sinus rhythm was present in 170 (78\%) patients and AF in 34 (16\%). Of those 34 patients, 18 had no previous history of AF. Pacemaker rhythm was present in five $(2.3 \%)$ patients. Ten (4.6\%) patients had other haemodynamically non-significant rhythm (five junctional rhythm, two nodal rhythm, two ventricular rhythm, and one supraventricular tachycardia). Each of the clinical signs of hypoperfusion was observed very frequently (Table 2). Left ventricular systolic function was impaired at baseline, with mean LVEF of $33 \%$ (14\%). The most common cause of shock was ACS $(81 \% ; n=177)$, with non-ACS causes accounting for the remaining $19 \%(n=42)$.

The majority of ACS patients ( $n=148 ; 68 \%$ of all patients) presented with STEMI whereas $19(9 \%)$ had a mechanical complication of $\mathrm{MI}$ including 6 ruptures of papillary muscle, 10 of ventricular septum and 3 of LV free wall. Non-ACS causes consisted mainly of worsening of chronic heart failure (11\%), valvular and other mechanical causes (6\%), stress-induced cardiomyopathy (Tako-Tsubo; $2 \%$ ), and myocarditis (2\%).

\section{Acute coronary syndrome and non-acute coronary syndrome causes of cardiogenic shock}

The characteristics and clinical picture of cardiogenic shock patients with and without ACS are compared in Tables 1 and 2. The prevalence of history of CAD or previous $\mathrm{MI}$ was similar in both groups. Patients with non-ACS, in whom previous history of heart failure was more frequent, had low TnT levels but significantly higher levels of NT-proBNP at baseline compared with ACS patients. Levels of TnT on admission were a good discriminator between patients with and without ACS (AUC 0.91, 95\% Cl 0.86-0.96; $P<0.001)$. Nevertheless, although non-ACS shock comprised a variety of aetiologies, the clinical presentation and medical treatment were very similar in both patients groups (Table 2; Supplementarty material online, Table S1). Subjects in the non-ACS subgroup were on average younger with a high proportion of women. AF was more common in non-ACS patients both in the medical history (see Table 2) and as rhythm at presentation ( $21 \%$ vs. $14 \%$ in ACS patients, $P=0.2$ ). Nevertheless, new-onset (no known history of) AF was actually observed more frequently in ACS patients $(n=17)$ compared with non-ACS patients $(n=1)$. Pneumonia was diagnosed in six (14\%) non-ACS patients, and other infections in five (12\%). Baseline LVEF was similar in both groups, but moderate to severe mitral regurgitation was observed in nearly a half of non-ACS shock patients. 
Table 2 Comparison of clinical presentation, biochemistry and mortality of all cardiogenic shock patients and of those with and without acute coronary syndromes

\begin{tabular}{|c|c|c|c|c|}
\hline Characteristic & All $(n=219)$ & $\operatorname{ACS}(n=177)$ & Non-ACS $(n=42)$ & $P$-value \\
\hline Systolic blood pressure, $\mathrm{mmHg}$ & $78(14)$ & $77(14)$ & $79(11)$ & 0.6 \\
\hline Diastolic blood pressure, $\mathrm{mmHg}$ & $47(10)$ & $46(11)$ & $48(9)$ & 0.3 \\
\hline Mean arterial pressure, $\mathrm{mmHg}$ & $57(11)$ & $56(11)$ & $59(9)$ & 0.14 \\
\hline Heart rate, b.p.m. & $90(28)$ & $89(29)$ & $96(24)$ & 0.1 \\
\hline Sinus rhythm & $170(78)$ & $140(79)$ & $30(71)$ & 0.3 \\
\hline \multicolumn{5}{|l|}{ Clinical findings, $n(\%)$} \\
\hline Cold periphery & $207(95)$ & $166(94)$ & $41(98)$ & 0.4 \\
\hline Confusion & $148(68)$ & $126(71)$ & $22(52)$ & 0.04 \\
\hline Oliguria & $121(55)$ & $98(55)$ & $24(57)$ & 0.7 \\
\hline Lactate $>2 \mathrm{mmol} / \mathrm{L}$ & $155(71)$ & $126(71)$ & $29(69)$ & 0.9 \\
\hline Resuscitated from cardiac arrest & $62(28)$ & $55(31)$ & $7(17)$ & 0.06 \\
\hline Time from detection of shock to study inclusion, min & $105(0-210)$ & $100(0-195)$ & $120(28-240)$ & 0.2 \\
\hline \multicolumn{5}{|l|}{ Baseline echocardiography } \\
\hline LVEDD (mm) & $52(9)$ & $51(8)$ & $59(11)$ & $<0.001$ \\
\hline LVEF (\%) & $33(14)$ & $34(14)$ & $30(15)$ & 0.12 \\
\hline LVEF $<40 \%$ & $135(65)$ & $107(63)$ & $28(72)$ & 0.3 \\
\hline Mitral regurgitation (moderate or severe), $n(\%)$ & $73(35)$ & $54(32)$ & $19(48)$ & 0.07 \\
\hline \multicolumn{5}{|l|}{ Biochemistry } \\
\hline Blood haemoglobin $(\mathrm{g} / \mathrm{L})$ & $128(22)$ & $129(22)$ & $127(21)$ & 0.7 \\
\hline Sodium (mmol/L) & $137(5)$ & $137(5)$ & $136(7)$ & 0.3 \\
\hline Potassium (mmol/L) & $4.2(0.8)$ & $4.2(0.8)$ & $4.4(0.9)$ & 0.3 \\
\hline Arterial blood lactate $(\mathrm{mmol} / \mathrm{L})$ & $2.8(1.7-5.8)$ & $3.0(1.8-5.8)$ & $2.6(1.3-5.8)$ & 0.3 \\
\hline Arterial blood pH & $7.30(7.20-7.40)$ & $7.30(7.20-7.40)$ & $7.30(7.20-7.40)$ & 0.2 \\
\hline hsTnT (ng/L) & $2190(388-5418)$ & $2873(1056-7555)$ & $104(40-389)$ & $<0.001$ \\
\hline NT-proBNP (pg/mL) & $2710(585-9434)$ & $1948(472-9093)$ & $6431(2522-14064)$ & 0.006 \\
\hline Creatinine $(\mathrm{mmol} / \mathrm{L})$ & $104(78-140)$ & 101 (79-139) & $111(64-162)$ & 0.8 \\
\hline eGFR $\left(\mathrm{mL} / \mathrm{min} / 1.73 \mathrm{~m}^{2}\right)$ & $61(41-87)$ & $61(42-86)$ & $61(32-97)$ & 0.8 \\
\hline $\operatorname{CRP}(g / L)$ & $16(4-54)$ & $13(4-48)$ & $29(7-91)$ & 0.03 \\
\hline In-hospital length of stay, days & $12(7-25)$ & $11(6-27)$ & $16(10-24)$ & 0.11 \\
\hline In-hospital mortality, $n(\%)$ & $80(37)$ & $70(40)$ & $10(24)$ & 0.06 \\
\hline
\end{tabular}

Data are presented as numbers and percentages (\%), mean (standard deviation), and median (IQR).

$P$-values are for the difference between ACS and non-ACS groups.

ACS, acute coronary syndrome; CRP, C-reactive protein; eGFR, estimated glomerular filtration rate by the Chronic Kidney Disease Epidemiology Collaboration formula hsTnT, highly sensitive troponin T; IQR, interquartile range; LVEDD, left ventricular end-diastolic diameter.

Management and procedures are detailed in Supplementarty material online, Table S1. Overall, $85 \%$ of patients received a vasopressor (norepinephrine, epinephrine, dopamine, vasopressin, or terlipressin) and $66 \%$ an inotrope (dobutamine, levosimendan, milrinone, or enoximone). The majority of patients received a vasopressor-inotrope combination. Only vasopressors were given to $29 \%$ of patients and only inotropes to $10 \%$. Rates of vasopressor and inotrope support were also comparable in ACS and non-ACS groups. Almost all patients (94\%) with ACS aetiology underwent coronary angiogram, and of these $89 \%$ underwent $\mathrm{PCl}$. Of the remaining $11 \%$, seven patients underwent surgical correction of mechanical complication, three had only CABG, and nine ACS patients were treated conservatively.

\section{Mortality and predictors of hospital death}

There were 70 deaths (32\%) in the intensive or cardiac care units, and a total of 80 deaths $(37 \%)$ during hospital stay. Non-ACS patients had a more favourable course compared with patients with ACS aetiology (Table 2). Univariate predictors of in-hospital mortality are shown in Supplementarty material online, Table S2. Adjusted ORs for factors associated with mortality are shown in Table 3. Interestingly, ACS was independently associated with worse prognosis (OR 7.4, 95\% Cl 1.9-29.8; $P=0.005$ ), suggesting clearly better survival in patients with other causes of cardiogenic shock. The Kaplan-Meier survival curve stratified by ACS aetiology is shown in Figure 1.

\section{The CardShock risk Score for prediction of in-hospital mortality}

Using the variables from Table 3, we created a prediction model for in-hospital mortality. The prediction model exhibited excellent discrimination, with an AUC of 0.85 (95\% Cl 0.79-0.90; $P<0.001)$ for hospital mortality compared with an AUC of $0.76(95 \% \mathrm{Cl}$ $0.69-0.83$ ) for the Sleeper score from the SHOCK trial (Figure 2). ${ }^{13}$ The prediction model was validated in the IAPB-SHOCK II trial 
Table 3 Predictors of in-hospital mortality in cardiogenic shock

\begin{tabular}{|c|c|c|}
\hline Variable & $\begin{array}{l}\text { Adjusted OR } \\
(95 \% \mathrm{Cl})\end{array}$ & $P$-value ${ }^{a}$ \\
\hline Prior CABG & $10.7(1.8-64.7)$ & 0.01 \\
\hline ACS aetiology & $7.4(1.9-29.8)$ & 0.005 \\
\hline Confusion & $3.0(1.1-8.1)$ & 0.03 \\
\hline $\begin{array}{l}\text { Previous myocardial } \\
\text { infarction }\end{array}$ & $3.2(1.2-8.2)$ & 0.02 \\
\hline Blood lactate (per mmol/L) & $1.4(1.2-1.6)$ & $<0.001$ \\
\hline LVEF (per \% decrease) & $1.06(1.02-1.09)$ & 0.001 \\
\hline Age (per year) & $1.04(1.00-1.08)$ & 0.08 \\
\hline $\begin{array}{l}\text { Systolic blood pressure (per } \\
\mathrm{mmHg} \text { decrease) }\end{array}$ & $1.03(0.99-1.06)$ & 0.09 \\
\hline
\end{tabular}

The multivariable model included all variables in the table and further contained gender and a variable accounting for possible differences between centres. ACS, acute coronary syndrome; CABG, coronary artery bypass grafting; $\mathrm{Cl}$, confidence interval; $O R$, odds ratio.

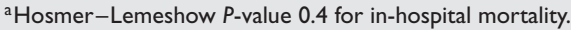

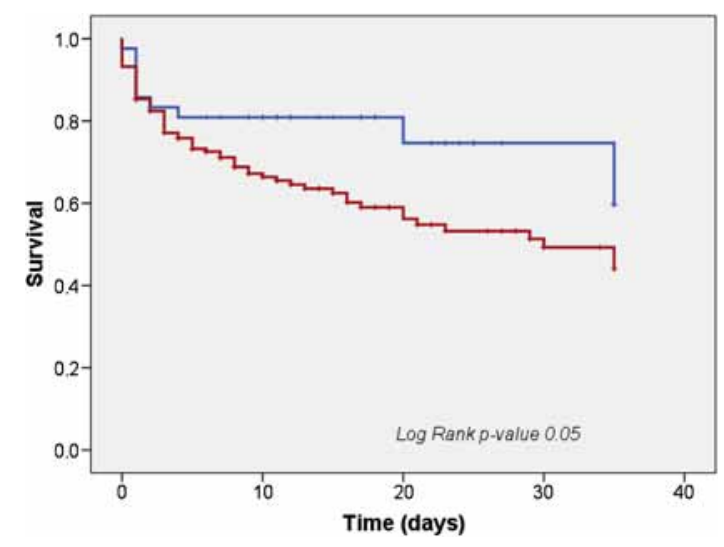

Figure 1 Kaplan-Meier in-hospital survival curves for cardiogenic shock patients with (red line) and without (blue line) acute coronary syndrome aetiology.

population and further refined to the CardShock risk Score which also included eGFR as a variable (Supplementarty material online, Appendix 1).

Finally, the CardShock risk Score consists of seven variables giving a maximum of nine points (Table 4). It had similar discrimination to the prediction model with an AUC of 0.85 (95\% Cl 0.80-0.90; $P<0.001$ ) (Figure 2; Supplementarty material online, Figure S1). For comparison, the APACHE II score ${ }^{14}$ had an AUC of $0.76(95 \%$ $\mathrm{Cl}$ 0.67-0.84) in the total CardShock population (Figure 2). In the validation cohort, the CardShock risk Score (AUC 0.71, 95\% $\mathrm{Cl}$ 0.66-0.76) also had better predictive performance than the Sleeper score (Figure 2). In addition, the CardShock risk Score performed well in both ACS (AUC 0.83, 95\% Cl 0.77-0.89) and non-ACS (AUC 0.94, 95\% Cl 0.87-1.0; $P<0.001$ for both) groups (Supplementarty material online, Figure S2). Again, in the ACS group, the AUC of the Sleeper score was 0.75 (95\% Cl 0.68-0.83) and that of the APACHE II score was $0.75(95 \% \mathrm{Cl} 0.66-0.84)$.

The distribution of the study population across cumulative points in risk score and the stepwise increase in both predicted and observed mortality with higher scores are shown in Figure 3. The population can be classified according to the CardShock risk Score into low (scores 0-3), intermediate (scores 4-5), and high (scores 6-9) risk groups with observed mortality of $8.7,36$ and $77 \%$, respectively.

\section{Discussion}

The CardShock study is by far the largest European prospective, observational multicentre and multinational study on cardiogenic shock, describing a contemporary cohort of unselected patients with cardiogenic shock covering a broad spectrum of aetiologies. STEMI and other forms of ACS are still the leading causes of cardiogenic shock. Almost all of these patients undergo coronary angiogram and percutaneous or surgical revascularization. Nevertheless, one-fifth of shock cases have an aetiology other than ACS. Interestingly, our study clearly suggests that despite having similar clinical presentation and severity, ACS aetiology is independently associated with increased mortality compared with non-ACS. Finally, the CardShock risk Score, consisting of seven easily recognizable clinical variables, shows good performance in early risk stratification and prediction of short-term mortality in cardiogenic shock.

For many patients, shock was the first manifestation of heart disease. Hypertension and diabetes were commonly present, but only a minority had previous ischaemic heart disease or a history of heart failure, as also described in other cohorts. ${ }^{4,9}$ Confusion and elevated blood lactate at presentation indicate the presence of severe systemic hypoperfusion and organ dysfunction, and were independently associated with higher risk of death in the present study. Still, the clinical significance of confusion is often underestimated in daily clinical practice. An increase in blood lactate is a well-known prognostic factor in critical illness. It derives from increased lactate production in hypoperfusion states or decreased lactate clearance that may also be secondary to liver dysfunction. Indeed, multiorgan damage and failure, or systemic pathways activated in the state of cardiogenic shock, have been suggested to contribute to the detrimental prognosis. ${ }^{15-17}$

While the literature is largely focused on cardiogenic shock secondary to $\mathrm{Ml}$, a significant proportion of reported cases have aetiologies other than ACS. These disorders are heterogeneous, ranging from advanced severe heart failure to temporary cardiac disturbances, sometimes correctable by interventions or even self-limiting. ${ }^{10,11}$ Consequently, other causes should be actively evaluated, especially in the absence of STEMI.

There are some interesting observations regarding management. Following the publication of the landmark SHOCK trial, angiography and early invasive revascularization became the preferred approach to shock caused by ACS. ${ }^{5}$ Moreover, it should be an unambiguous goal to avoid prolonged hypoperfusion and development of multiorgan injury and failure. Vasoactive medications and 


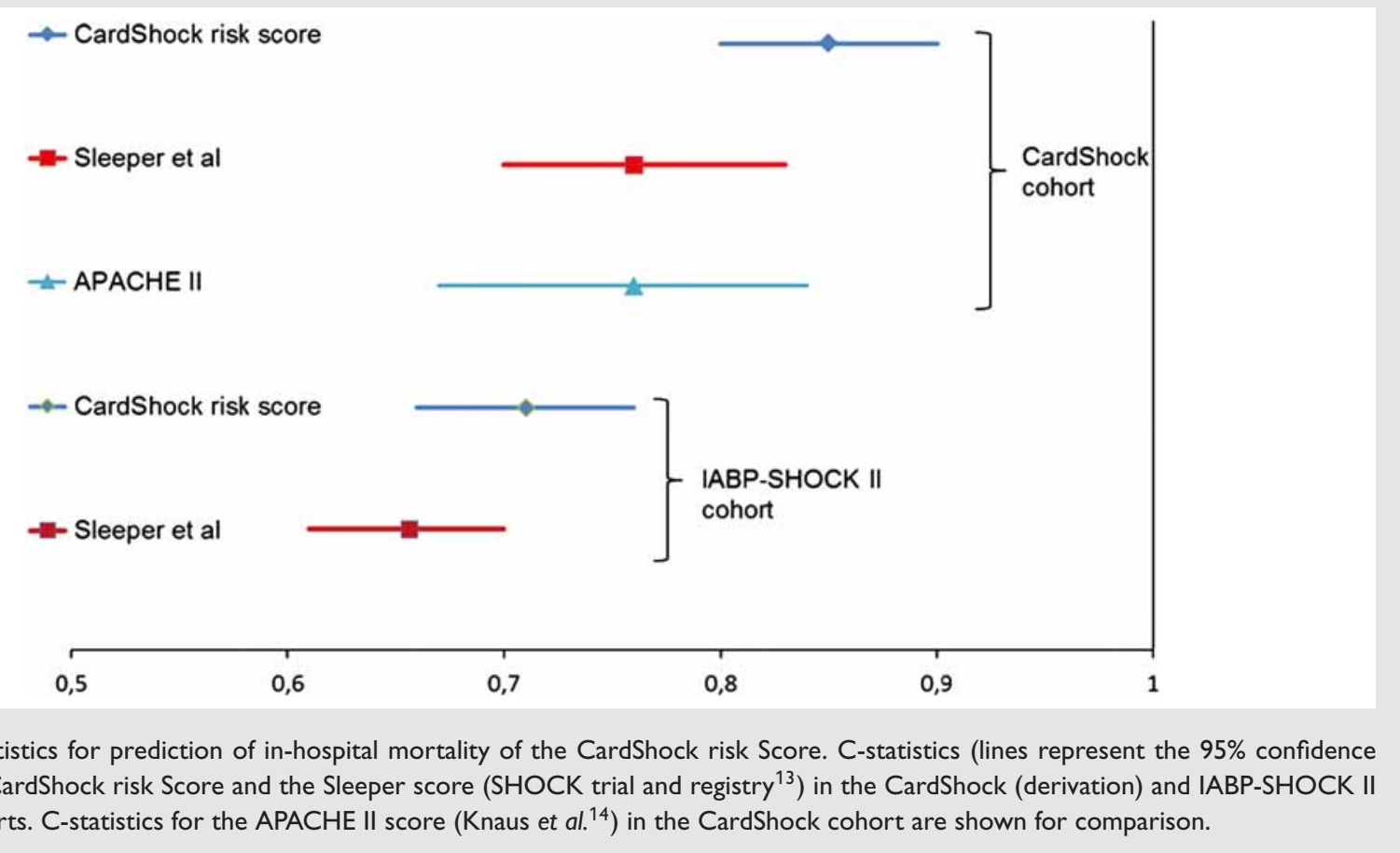

Table 4 The CardShock risk Score for risk prediction of in-hospital mortality in cardiogenic shock

\begin{tabular}{lc}
\hline Variable & CardShock risk Score \\
$\ldots \ldots \ldots \ldots \ldots \ldots \ldots \ldots \ldots \ldots \ldots \ldots \ldots \ldots \ldots \ldots \ldots \ldots$ \\
Age $>75$ years & 1 \\
Confusion at presentation & 1 \\
Previous Ml or CABG & 1 \\
ACS aetiology & 1 \\
LVEF $<40 \%$ & 1 \\
Blood lactate & \\
$<2 \mathrm{mmol} / \mathrm{L}$ & 0 \\
$2-4 \mathrm{mmol} / \mathrm{L}$ & 1 \\
$>4 \mathrm{mmol} / \mathrm{L}$ & 2 \\
eGFR $\mathrm{CKD}-\mathrm{EPI}$ & \\
$>60 \mathrm{~mL} / \mathrm{min} / 1.73 \mathrm{~m}^{2}$ & 0 \\
$30-60 \mathrm{~mL} / \mathrm{min} / 1.73 \mathrm{~m}^{2}$ & \\
$<30 \mathrm{~mL} / \mathrm{min} / 1.73 \mathrm{~m}^{2}$ & 2 \\
Maximum points & 9 \\
\hline
\end{tabular}

ACS, acute coronary syndrome; CABG, coronary artery bypass grafting; MI, myocardial infarction; eGFR ${ }_{\text {CKD-EPI }}$, estimated glomerular filtration rate by the Chronic Kidney Disease Epidemiology Collaboration formula.

inotropes are still required in most patients with severe circulatory shock, although their benefit is uncertain, with concerns about safety. ${ }^{15,18,19}$

Intra-aortic balloon support was still rather common in the present study. However, the IABP-SHOCK II trial which showed the lack of survival benefit of intra-aortic balloon treatment in MI complicated by shock was published shortly before the end of the recruitment of the present study. ${ }^{9}$ The infrequent use of ventricular assist devices perhaps reflects difficulty in patient selection. Considering the current high mortality rate, early selection of patients for advanced management including rational use of mechanical assist devices seems plausible, but remains to be proven.

The short-term mortality of $\sim 40 \%$ in the present study is in line with that in the recent IABP-SHOCK II trial. ${ }^{9}$ Improving survival has been observed during the past two decades, attributed to the introduction of routine use of percutaneous revascularization in STEMI and modern intensive care. ${ }^{2,3,20}$ Nevertheless, survival in cardiogenic shock is still dismal.

Acute coronary syndrome was an independent predictor of in-hospital mortality despite very high rates of revascularization procedures. Since aetiologies other than ACS have been excluded from most studies of cardiogenic shock, this finding is, to our knowledge, novel. ACS with shock is associated with irreversible myocardial damage of significant magnitude, which often triggers inflammatory and other systemic responses. Previous MI or CABG, which may reflect a more extensive and severe ischaemic heart disease and limited reserves for recovery, were also strongly predictive of poor outcome. Interestingly, the clinical presentation, the prevalence of previous CAD, as well as mean LVEF were similar between patients with and without ACS.

The CardShock risk Score incorporated common clinical variables for prediction of in-hospital mortality. These parameters are readily available from clinical assessment, and the score can be calculated within a very short time after presentation of shock, which facilitates early risk stratification. From the SHOCK trial and registry data, Sleeper et al. developed a scoring system with clinical predictors of short-term outcome. ${ }^{13}$ Several of the variables in this scoring system are also found in our model, i.e. older age, signs of peripheral hypoperfusion, cerebral dysfunction 

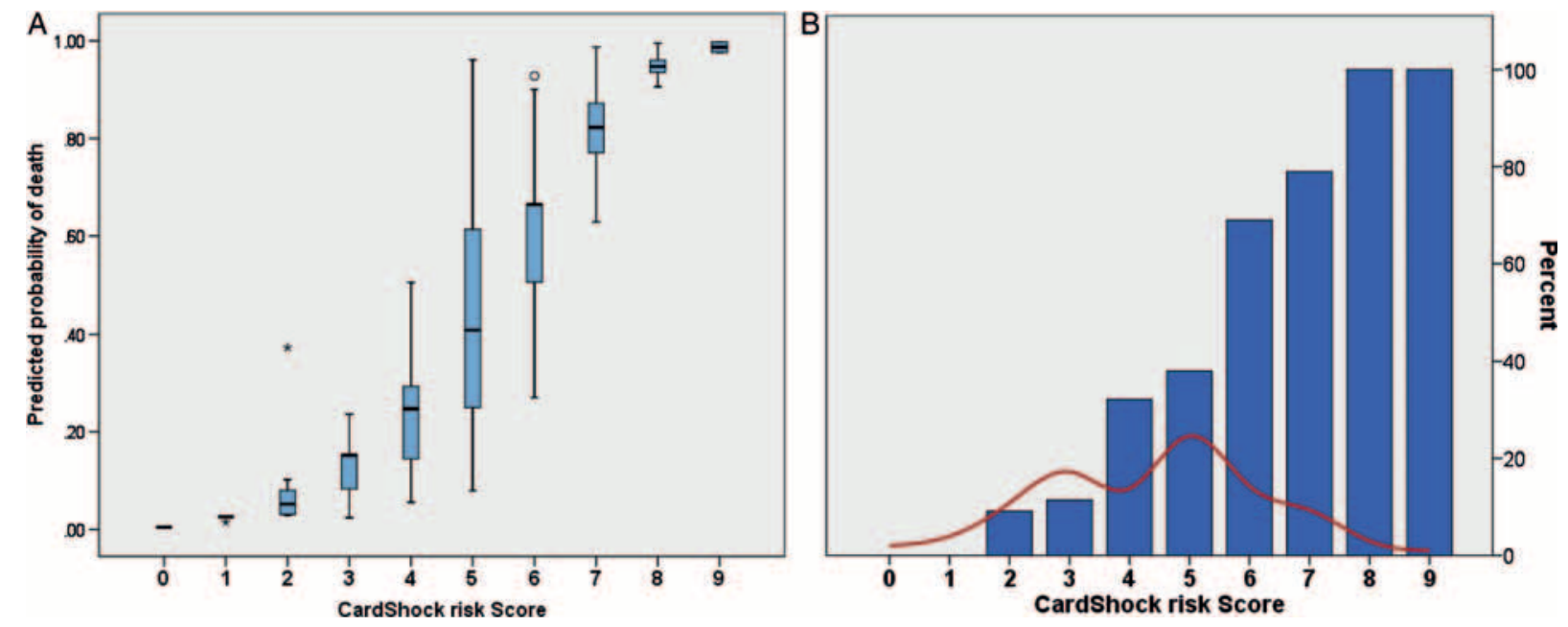

Figure 3 The CardShock risk Score. (A) Predicted risk of death with the CardShock risk Score. Boxplots show the median with the interquartile range of calculated predicted risk of death in each of the CardShock risk categories. Lines constitute the 10th and 90th percentile. The asterisk and circle are outliers. The $x$-axis depicts the cumulative points from the CardShock risk Score and the $y$-axis the predicted risk from the multivariable model. (B) Distribution of the population (red line) and in-hospital mortality (\%; blue bars) according to the cumulative points from the CardShock risk Score. The population can be classified according to the CardShock risk Score into low (scores 0-3), intermediate (scores 4-5), and high (scores 6-9) risk groups with observed mortalities of $8.7,36$, and $77 \%$, respectively.

(though defined as a much more severe form, i.e. anoxic brain injury in the SHOCK trial), LVEF, and prior CABG. ${ }^{13}$ However, arterial blood $\mathrm{pH}$ was not an independent predictor of risk. The independent associations of elevated blood lactate and altered mental status with mortality in the IABP-SHOCK II trial emphasize the importance of hypoperfusion for prognosis. ${ }^{21}$ Prior $\mathrm{MI}$ or CABG were not associated with outcome in IABP-SHOCK II, but the prevalence was low. Incorporating a measure of renal function in the risk prediction model seems clinically justified and improved the performance of the score in the validation cohort. ${ }^{13,21-23}$ Severity of LV systolic dysfunction also correlates with outcome in cardiogenic shock. ${ }^{21,24}$ Indeed, echocardiography is clearly indicated in every patient with shock to assess ventricular and valvular function and to detect mechanical complications. In contrast to what has been described in other populations of critically ill patients, heart rate was not a predictor of risk in this population. ${ }^{25}$

In everyday clinical practice, older age is often an exclusion criterion for advanced therapies such as prolonged intensive care, cardiac transplantation, or the use of mechanical assist devices. However, in light of our data, though older age increases early mortality, it is just one of the predictors of outcome. Thus, in addition to age, both medical history and several factors on admission have to be included in individual decision-making.

A central aim of the study was to develop a practical tool for risk assessment of short-term mortality for patients with cardiogenic shock. Prognostic risk scores for unselected acute heart failure populations cannot be applied to cardiogenic shock, which represents only $2-4 \%$ of cases with acute heart failure. Scores assessing organ dysfunction and disease severity in the critically ill (APACHE, SAPS, MODS, and SOFA) have been shown also to predict mortality in patients with cardiogenic shock, but were primarily developed for assessing disease severity in patients admitted to the Intensive Care Unit (ICU). ${ }^{16,17}$ Moreover, in this study, the predictive performance of APACHE II was similar to the Sleeper score and poorer than the novel CardShock risk Score.

The contemporary CardShock risk Score is easy to assess, simple to calculate, and shows good performance for risk prediction of short-term mortality, both in the original CardShock and in the validation cohort. The risk score demonstrated good discrimination of poor outcome in both ACS and non-ACS patients. This ideally makes the score useful also for patients with severe forms of cardiomyopathies. In addition, in ACS patients, neither the APACHE II score nor the Sleeper score performed as well as the CardShock risk Score. The results show the strength and utility of the CardShock risk Score, which classifies patients in risk categories, with a clear and stepwise increase in mortality for the majority of the population with cardiogenic shock. We believe that the risk score might aid in selection of management strategies. Proper risk categorization may also be of importance in planning treatment trials to identify optimally patients that might benefit from current and novel therapies.

When the APACHE II score was introduced in 1985, it was advocated to be combined with an accurate description of disease and thereby to be used for prognostic stratification of a wide range of ill patients and for assisting investigators comparing the success of new or differing forms of therapy. ${ }^{14}$ The CardShock risk Score can have similar applications.

The aetiology, clinical presentation, and management of cardiogenic shock differ in several ways from those of a mixed ICU population. Thus, it seems warranted to have a specific risk score for patients with cardiogenic shock. The CardShock risk Score is a 
simple tool for classification of patients with clearly different mortality risks. In comparison, the score from the SHOCK trial is more complex to calculate, and was developed from a cohort recruited almost 20 years ago. ${ }^{13}$ As populations change and new diagnostic, therapeutic, and prognostic techniques become available, scoring systems need to be updated. ${ }^{26}$

The role of a risk score can be approached from several distinct views. In epidemiological studies, the CardShock risk Score would give a uniform description of severity and mortality risk across the population. Secondly, the absence of evidence-based therapies in cardiogenic shock is striking. Even most of the current therapies would need to be proven safe and effective. From the regulatory and clinical aspects, appropriate patient selection for randomized trials is crucial. In order to recruit a more homogenous population, patients have to be categorized according to the inherent risk of the syndrome. In a putative treatment trial, the CardShock risk Score could be used for targeting the study to include patients with intermediate to high, but not low or extremely high, mortality risk. Thirdly, it is obvious that novel, costly therapies with potentially high complication rates need to be targeted at pre-specified populations with a certain risk profile in order to achieve most benefit and cost-effectiveness. Finally, a systematic characterization of the population would enable benchmarking of different facilities as well as cost-effectiveness analyses. A score allows more uniform comparison of management strategies between different centres and countries.

There are some limitations that should be acknowledged. For a prospective cohort in cardiogenic shock, the CardShock study included a reasonable number of patients, but the statistical $\mathrm{Cls}$ are sometimes wide. Since most patients had ACS aetiology, the non-ACS group is heterogeneous and of limited size. There was no adjudication of the diagnosis and aetiology of cardiogenic shock. However, there were unambiguous clinical criteria for cardiogenic shock, and ACS was defined using current diagnostic criteria including electrocardiographic changes in patients with STEMI. In addition, angiographic findings were available in most of the patients for further confirmation of the aetiology though a centralized analysis of coronary angiograms was not performed for the present analysis. In older studies, the cardiac index and pulmonary artery wedge pressure were elemental for the definition of cardiogenic shock. ${ }^{5}$ However, in the present study only 39 patients $(17 \%)$ had a pulmonary artery catheter at baseline, while in some patients it was inserted only later. This limits the comparability of our results with the oldest studies. Validation of the CardShock risk Score was performed in a large contemporary population that included only ACS patients. A larger prospective validation cohort of unselected cardiogenic shock patients including a higher number of non-ACS cases would have been of great value. However, such a cohort is not currently available.

\section{Conclusion}

Cardiogenic shock is a state of severe systemic hypoperfusion due to cardiac failure characterized by haemodynamic compromise and a common need for vasoactive medication, and is still associated with high in-hospital mortality. While STEMI or mechanical complications of $\mathrm{Ml}$ are the most common causes of cardiogenic shock, $20 \%$ of patients have non-ACS aetiology. Despite current active use of early invasive revascularization, shock caused by ACS is associated with significantly higher mortality risk compared with other causes of cardiogenic shock. The CardShock risk Score incorporating seven easily available variables shows good discrimination and can be used for early prediction of in-hospital mortality. By objectively categorizing patients into discrete risk groups the score might aid in clinical decision-making, patient selection in clinical trials, as well as comparing the case mix of different patient populations.

\section{Acknowledgements}

The CardShock steering committee: Veli-Pekka Harjola (chair), Marek Banaszewski, Lars Kober, Johan Lassus, Alexandre Mebazaa, Marco Metra, John Parissis, Jose Silva-Cardoso, Alessandro Sionis, Salvatore Di Somma, Jindrich Spinar.

List of investigators: Athens: Katerina Koniari, Astrinos Voumvourakis, Apostolos Karavidas; Barcelona: Jordi Sans-Rosello, Montserrat Vila, Albert Duran-Cambra; Brescia: Marco Metra, Michela Bulgari, Valentina Lazzarini; Brno: Jiri Parenica, Roman Stipal, Ondrej Ludka, Marie Palsuva, Eva Ganovska, Petr Kubena; Copenhagen: Matias G. Lindholm, Christian Hassager; Helsinki: Tom Bäcklund, Raija Jurkko, Kristiina Järvinen, Tuomo Nieminen, Kari Pulkki, Leena Soininen, Reijo Sund, Ilkka Tierala, Jukka Tolonen, Marjut Varpula, Tuomas Korva, Mervi Pietilä, Anne Pitkälä; Rome: Rossella Marino; Porto: Alexandra Sousa, Carla Sousa, Mariana Paiva, Inês Rangel, Rui Almeida, Teresa Pinho, Maria Júlia Maciel; Warsaw: Janina Stepinska, Anna Skrobisz, Piotr Góral

The GREAT network (www.greatnetwork.org).

\section{Supplementary Information}

Additional Supporting Information may be found in the online version of this article:

Supplementary appendix 1

Figure S1 Area under the curve for the CardShock risk Score, model and the Sleeper score in the CardShock population

Figure S2 Performance of the CardShock risk Score in ACS and non-ACS subgroups

Table S1 Management of patients with cardiogenic shock

Table S2 Univariate predictors of in-hospital mortality in cardiogenic shock

Table S3 Comparison of the CardShock risk model with the CardShock risk Score

\section{Funding}

The CardShock study was supported by grants from Aarne Koskelo Foundation and the Finnish Cardiac foundation. Laboratory kits for analysis of NT-proBNP and hsTnT were kindly provided by Roche Diagnostics, Basel, Switzerland. 
Conflict of interest: V.-P.H. has served on advisory boards for Bayer, BMS/Pfizer, Boehringer-Ingelheim, Roche Diagnostics, Novartis, and Servier, and received lecture fees from Bayer, Orion Pharma, Resmed, and Roche Diagnostics. J.L. has served on advisory boards for Roche Diagnostics, Novartis, Servier, and Vifor Pharma, and received lecture fees from Bayer, Novartis, Orion Pharma, Pfizer, Resmed, and Roche Diagnostics. J.P. has received honoraria for lectures and advisory board meetings from Orion Pharma and Novartis. A.M. has served on advisory boards for Bayer, Cardiorentis, and The Medicines Company, and has received consulting fees from Pronota, and lecture fees from Alere, Edwards, Novartis, Orion, Vifor, and Thermofisher. A.M.'s research institution has a financial contract with Pronota. All other authors have no conflicts to declare,

\section{References}

1. Zeymer U, Vogt A, Zahn R, Weber MA, Tebbe U, Gottwik M, Bonzel T, Senges J, Neuhaus KL, Arbeitsgemeinschaft Leitende Kardiologische Krankenhausarzte (ALKK). Predictors of in-hospital mortality in 1333 patients with acute myocardial infarction complicated by cardiogenic shock treated with primary percutaneous coronary intervention $(\mathrm{PCl})$ : results of the primary $\mathrm{PCl}$ registry of the Arbeitsgemeinschaft Leitende Kardiologische Krankenhausarzte (ALKK). Eur Heart J 2004;25:322-328.

2. Jeger RV, Radovanovic D, Hunziker PR, Pfisterer ME, Stauffer JC, Erne P, Urban P, AMIS Plus Registry Investigators. Ten-year trends in the incidence and treatment of cardiogenic shock. Ann Intern Med 2008;149:618-626.

3. Goldberg RJ, Spencer FA, Gore JM, Lessard D, Yarzebski J. Thirty-year trends (1975 to 2005) in the magnitude of, management of, and hospital death rates associated with cardiogenic shock in patients with acute myocardial infarction: a population-based perspective. Circulation 2009;119:1211-1219.

4. Aissaoui N, Puymirat E, Tabone X, Charbonnier B, Schiele F, Lefevre T, Durand E, Blanchard D, Simon T, Cambou JP, Danchin N. Improved outcome of cardiogenic shock at the acute stage of myocardial infarction: a report from the USIK 1995, USIC 2000, and FAST-MI French nationwide registries. Eur Heart J 2012;33:2535-2543.

5. Hochman JS, Sleeper LA, Webb JG, Sanborn TA, White HD, Talley JD, Buller CE, Jacobs AK, Slater JN, Col J, McKinlay SM, Lejemtel TH. Early revascularization in acute myocardial infarction complicated by cardiogenic shock. SHOCK Investigators. Should we emergently revascularize occluded coronaries for cardiogenic shock. N Engl J Med 1999;341:625-634.

6. Task Force on the management of ST-segment elevation acute myocardial infarction of the European Society of Cardiology (ESC), Steg PG, James SK, Atar D, Badano LP, Blomstrom-Lundqvist C, Borger MA, Di Mario C, Dickstein K, Ducrocq G, Fernandez-Aviles F, Gershlick AH, Giannuzzi P, Halvorsen S, Huber $K$, Juni P, Kastrati A, Knuuti J, Lenzen MJ, Mahaffey KW, Valgimigli M, van 't Hof A, Widimsky P, Zahger D. ESC Guidelines for the management of acute myocardial infarction in patients presenting with ST-segment elevation. Eur Heart J 2012;33:2569-2619.

7. Authors/Task Force members, Windecker S, Kolh P, Alfonso F, Collet JP, Cremer J, Falk V, Filippatos G, Hamm C, Head SJ, Juni P, Kappetein AP, Kastrati A, Knuuti J, Landmesser U, Laufer G, Neumann FJ, Richter DJ, Schauerte P, Sousa Uva M, Stefanini GG, Taggart DP, Torracca L, Valgimigli M, Wijns W, Witkowski A, Authors/Task Force members. 2014 ESC/EACTS Guidelines on myocardial revascularization: The Task Force on Myocardial Revascularization of the European Society of Cardiology (ESC) and the European Association for Cardio-Thoracic Surgery (EACTS). Developed with the special contribution of the European Association of Percutaneous Cardiovascular Interventions (EAPCI). Eur Heart J 2014;35:2541-2619.

8. McMurray JJ, Adamopoulos S, Anker SD, Auricchio A, Bohm M, Dickstein K, Falk V, Filippatos G, Fonseca C, Gomez-Sanchez MA, Jaarsma T, Kober L, Lip GY, Maggioni AP, Parkhomenko A, Pieske BM, Popescu BA, Ronnevik PK, Rutten FH, Schwitter J, Seferovic P, Stepinska J, Trindade PT, Voors AA, Zannad F, Zeiher A, Bax JJ, Baumgartner H, Ceconi C, Dean V, Deaton C, Fagard R, Funck-Brentano C, Hasdai D, Hoes A, Kirchhof P, Knuuti J, Kolh P, McDonagh T, Moulin C, Reiner Z, Sechtem U, Sirnes PA, Tendera M, Torbicki A, Vahanian A, Windecker S, Bonet LA, Avraamides P, Ben Lamin HA, Brignole M, Coca A, Cowburn P, Dargie H, Elliott P, Flachskampf FA, Guida GF, Hardman S, lung B, Merkely B, Mueller C, Nanas JN, Nielsen OW, Orn S, Parissis JT, Ponikowski P. ESC guidelines for the diagnosis and treatment of acute and chronic heart failure 2012: the Task Force for the Diagnosis and Treatment of Acute and Chronic Heart Failure 2012 of the European Society of Cardiology. Developed in collaboration with the Heart Failure Association (HFA) of the ESC. Eur J Heart Fail 2012;14:803-869.

9. Thiele H, Zeymer U, Neumann FJ, Ferenc M, Olbrich HG, Hausleiter J, Richardt G, Hennersdorf M, Empen K, Fuernau G, Desch S, Eitel I, Hambrecht R, Fuhrmann J, Bohm M, Ebelt H, Schneider S, Schuler G, Werdan K, IABP-SHOCK II Trial Investigators. Intraaortic balloon support for myocardial infarction with cardiogenic shock. N Engl J Med 2012;367:1287-1296.

10. Topalian S, Ginsberg F, Parrillo JE. Cardiogenic shock. Crit Care Med 2008;36:S66-S74.

11. Gowda RM, Fox JT, Khan IA. Cardiogenic shock: basics and clinical considerations. Int J Cardiol 2008;123:221-228.

12. Levey AS, Stevens LA, Schmid CH, Zhang YL, Castro AF 3rd, Feldman HI, Kusek JW, Eggers P, Van Lente F, Greene T, Coresh J, CKD-EPI (Chronic Kidney Disease Epidemiology Collaboration). A new equation to estimate glomerular filtration rate. Ann Intern Med 2009;150:604-612.

13. Sleeper LA, Reynolds HR, White HD, Webb JG, Dzavik V, Hochman JS. A severity scoring system for risk assessment of patients with cardiogenic shock: a report from the SHOCK Trial and Registry. Am Heart J 2010;160:443-450.

14. Knaus WA, Draper EA, Wagner DP, Zimmerman JE. APACHE II: a severity of disease classification system. Crit Care Med 1985;13:818-829.

15. Reynolds HR, Hochman JS. Cardiogenic shock: current concepts and improving outcomes. Circulation 2008;117:686-697.

16. Kellner P, Prondzinsky R, Pallmann L, Siegmann S, Unverzagt S, Lemm H, Dietz S, Soukup J, Werdan K, Buerke M. Predictive value of outcome scores in patients suffering from cardiogenic shock complicating AMI: APACHE II, APACHE III, Elebute-Stoner, SOFA, and SAPS II. Med Klin Intensivmed Notfmed 2013;108:666-674.

17. Prondzinsky R, Lemm H, Swyter M, Wegener N, Unverzagt S, Carter JM, Russ M, Schlitt A, Buerke U, Christoph A, Schmidt H, Winkler M, Thiery J, Werdan K, Buerke M. Intra-aortic balloon counterpulsation in patients with acute myocardial infarction complicated by cardiogenic shock: the prospective, randomized IABP SHOCK Trial for attenuation of multiorgan dysfunction syndrome. Crit Care Med 2010;38:152-160.

18. De Backer D, Biston P, Devriendt J, Madl C, Chochrad D, Aldecoa C, Brasseur A, Defrance P, Gottignies P, Vincent JL, SOAP II Investigators. Comparison of dopamine and norepinephrine in the treatment of shock. N Engl J Med 2010;362:779-789.

19. Levy B, Perez P, Perny J, Thivilier C, Gerard A. Comparison of norepinephrine-dobutamine to epinephrine for hemodynamics, lactate metabolism, and organ function variables in cardiogenic shock. A prospective, randomized pilot study. Crit Care Med 2011;39:450-455.

20. Zannad F, Mebazaa A, Juilliere Y, Cohen-Solal A, Guize L, Alla F, Rouge P, Blin P, Barlet MH, Paolozzi L, Vincent C, Desnos M, Samii K, for the EFICA Investigators. Clinical profile, contemporary management and one-year mortality in patients with severe acute heart failure syndromes: the EFICA study. Eur J Heart Fail 2006;8:697-705.

21. Thiele H, Zeymer U, Neumann FJ, Ferenc M, Olbrich HG, Hausleiter J, de Waha A, Richardt G, Hennersdorf M, Empen K, Fuernau G, Desch S, Eitel I, Hambrecht R, Lauer B, Bohm M, Ebelt H, Schneider S, Werdan K, Schuler G, on behalf of the Intraaortic Balloon Pump in cardiogenic shock II (IABP-SHOCK II) trial investigators. Intra-aortic balloon counterpulsation in acute myocardial infarction complicated by cardiogenic shock (IABP-SHOCK II): final 12 month results of a randomised, open-label trial. Lancet 2013;382:1638-1645.

22. Klein LW, Shaw RE, Krone RJ, Brindis RG, Anderson HV, Block PC, McKay CR, Hewitt K, Weintraub WS, American College of Cardiology National Cardiovascular Data Registry. Mortality after emergent percutaneous coronary intervention in cardiogenic shock secondary to acute myocardial infarction and usefulness of a mortality prediction model. Am J Cardiol 2005;96:35-41.

23. Katz JN, Stebbins AL, Alexander JH, Reynolds HR, Pieper KS, Ruzyllo W, Werdan K, Geppert A, Dzavik V, Van de Werf F, Hochman JS, TRIUMPH Investigators. Predictors of 30-day mortality in patients with refractory cardiogenic shock following acute myocardial infarction despite a patent infarct artery. Am Heart J 2009;158:680-687.

24. Garcia-Alvarez A, Arzamendi D, Loma-Osorio P, Kiamco R, Masotti M, Sionis A, Betriu A, Brugada J, Bosch X. Early risk stratification of patients with cardiogenic shock complicating acute myocardial infarction who undergo percutaneous coronary intervention. Am J Cardiol 2009;103:1073-1077.

25. Sander O, Welters ID, Foëx P, Sear JW. Impact of prolonged elevated heart rate on incidence of major cardiac events in critically ill patients with a high risk of cardiac complications. Crit Care Med 2005;33:81-88.

26. Vincent JL, Moreno R. Clinical review: scoring systems in the critically ill. Crit Care 2010;14:207. 